\title{
Identificação Imuno-histoquimica de Listeria monocytogenes em Placentas Fixadas em Formol e Embebidas em Parafina
}

\author{
Immunohistochemical Identification of Listeria monocytogenes in Formalin-fixed \\ and Paraffin-embedded Placentas \\ Jussara Pires Schwab, Maria Isabel Albano Edelweiss
}

\begin{abstract}
RESUMO
Objetivos: identificar Listeria monocytogenes (Lm) em placentas humanas pela técnica de imuno-histoquimica (IHQ) e relacionar sua presença com as alterações histológicas encontradas no exame convencional, com o trimestre gestacional, a idade das gestantes, casos de aborto e parto prematuro e a ocorrência de aborto habitual.

Métodos: um estudo retrospectivo foi realizado no setor de patologia de um hospital-escola de Porto Alegre no ano 2000. O material dos blocos de parafina de 254 placentas (exames anatomopatológicos), provenientes de aborto, de parto prematuro e de nascimento a termo, foi analisado pela técnica histológica convencional com a coloração de hematoxilina e eosina (HE). A técnica de IHQ foi realizada no material de 148 exames anatomopatológicos, que apresentaram alterações inflamatórias, hemorragia, necrose e trombose, utilizando anticorpo policlonal Rabbit A “Listeria monocytogenes" B65420R (Biodesign ${ }^{\circledR}$ ) na diluição 1:1000 e complexo avidina-biotina-estreptavidina. $O$ teste $\chi^{2}$ foi aplicado para a análise estatistica. Resultados: a presença de Lm foi identificada em 33,7\% das placentas analisadas pela técnica IHQ. Corioamnionite e vilite foram as alterações inflamatórias que estiverem associadas a diferença significativa nas placentas positivas. Lm esteve presente nas placentas de $1^{\circ}, 2^{\circ}$ e $3^{\circ}$ trimestre gestacional. Não houve associação entre idade das gestantes, casos de aborto e/ ou parto prematuro e a presença ou ausência de Lm nas placentas. Abortos habituais ocorreram em pacientes com ou sem Lm no tecido placentário.

Conclusão: a técnica de IHQ pode ser utilizada para confirmar o diagnóstico histopatológico de listeriose em todos os trimestres gestacionais.
\end{abstract}

PALAVRAS-CHAVES: Placenta. Listeria monocytogenes. Aborto habitual. Corioamnionite.

\section{Introdução}

A listeriose é zoonose causada pelo microrganismo Listeria monocytogenes $(\mathrm{Lm})$ e pode ser transmitida ao homem pelos alimentos de origem

Faculdade de Veterinária e Faculdade de Medicina da Universidade Federal do Rio Grande do Sul e Hospital de Clinicas de Porto Alegre. Porto Alegre/RS.

Trabalho realizado no Laboratório de Patologia Experimental do Hospital de Clínicas de Porto Alegre (HCPA)

Correspondência:

Jussara Pires Schwab

Rua Cel. Bordini, 1180, apto. 31 - Bairro Moinhos de Vento 90440-003 - Porto Alegre - RS

Fone: (51) 3333-2507 / Fax: (051) 3332-7246

e-mail jpschwab@orion.ufrgs.br

Apoio financeiro parcial do Fundo de Incentivo à Pesquisa e Eventos do Hospital de Clínicas de Porto Alegre animal, como o leite sem pasteurização, carne e os derivados de ambos ${ }^{1}$. Os indivíduos com sistema imunológico debilitado, gestantes e recém-nascidos fazem parte do grupo de risco e devem consumir esses alimentos com a necessária cautela ${ }^{2,3}$.

Ao longo do tempo, vários surtos de listeriose humana ocorreram, atingindo principalmente mulheres grávidas e seus conceptos, ocasionando aborto ou parto prematuro com alta incidência de mortes fetais e neonatais. Casos isolados de listeriose também provocaram aborto ou o nascimento de crianças com meningite e outras lesões neurológicas ${ }^{4-7}$.

A incidência de listeriose é subestimada, pois há vários fatores que dificultam a identificação do microrganismo por meio da cultura do san- 
gue e do líquido cefalorraquidiano, entre os quais citamos as características morfológicas da $\mathrm{Lm}$, que podem se confundidas com as de outro microrganismo, Streptococcus do grupo $\mathrm{B}^{8}$.

A listeriose não é, na maioria das vezes, diagnosticada na gravidez pela dificuldade das técnicas microbiológicas em encontrar o microrganismo ou pelas alterações histológicas nas placentas, o que a confunde com outras doenças, o que provavelmente impede de avaliar a importância de $L m$ na saúde humana ${ }^{9}$.

No entanto, é importante diagnosticá-la, por meio do exame da placenta, para prevenir processos inflamatórios maternos recidivantes e tratar os casos neonatais, que podem causar meningite com seqüelas como hidrocefalia, retardo mental, convulsões e sinais neurológicos focais ${ }^{10}$.

A ocorrência de aborto habitual ou de repetição (3 ou mais abortos consecutivos) relacionado com a presença de $L m$ é discutida por vários autores e não é investigada na maioria desses casos ${ }^{11}$.

A identificação de $\mathrm{Lm}$, pelo exame imunohistoquímico (IHQ), foi evidenciada em estudos de placentas que apresentaram as lesões inflamatórias características, o que demonstra ser método capaz de confirmar o diagnóstico desta enfermidade ${ }^{11,12}$.

Os objetivos deste trabalho foram identificar Lm em placentas humanas pela técnica de IHQ e relacionar sua presença com as alterações histológicas encontradas no exame convencional (HE), o trimestre gestacional, a idade das gestantes, os casos de aborto e parto prematuro e a ocorrência de aborto habitual.

\section{Material e Métodos}

Estudo retrospectivo feito a partir dos exames anatomopatológicos (AP) de placentas provenientes de aborto, parto prematuro e nascimento a termo. O levantamento efetuado nos registros do Serviço de Patologia do Hospital de Clínicas de Porto Alegre (HCPA) revelou 714 AP desse tipo de material examinados no ano 2000. Uma amostra casual de 254 AP foi utilizada para análise por meio do exame microscópico convencional.

O critério de leitura das lâminas, coradas com hematoxilina e eosina (HE), levou em consideração os achados histológicos, evidenciados pelo infiltrado inflamatório, hemorragia, necrose e trombose, observados ao microscópio nas objetivas 10, 40 e 100.

A técnica de IHQ foi realizada no material dos blocos de parafina de 148 lâminas examinadas pelo $\mathrm{HE}$ que apresentaram infiltrado inflamatório, hemorragia, necrose e trombose entre os 254 AP da amostra.
O método de IHQ para diagnóstico de listeriose foi padronizado no laboratório de patologia experimental do $\mathrm{HCPA}^{12}$ a partir da técnica de Santos et al. ${ }^{13}$, utilizando anticorpo primário policlonal Rabbit A "Listeria monocytogenes" B65420R (Biodesign ${ }^{\circledR}$ ) na diluição 1:1000 e complexo avidina-biotina-estreptavidina.

Os passos da técnica são: preparação dos cortes histológicos; desparafinização e hidratação; recuperação antigênica; irradiação em forno de microondas para bloqueio da peroxidase endógena; as lâminas foram colocadas em solução de peróxido de hidrogênio a 5\% e água destilada. Após o bloqueio em solução a $5 \%$ de leite desnatado e tampão fosfato (PBS - Dako ${ }^{\circledR}$ e 40 min em câmara úmida, foram lavadas com água corrente, água destilada e colocadas no PBS durante $5 \mathrm{~min}$; incubação com anticorpo primário policlonal Rabbit $A$ "Listeria monocytogenes" B65420R (Biodesign ${ }^{\circledR}$ ) na diluição 1:1000; foi feita a reação complexo avidinabiotina-estreptavidina + Kit Peroxidase: anticorpo secundário e marcador (Kit Lsab - Dako ${ }^{\circledR}$ ); revelação (Kit Dab - Dako ${ }^{\circledR}$ ); contracoloração (hematoxilina de Harris).

O critério para a leitura das lâminas, coradas pela IHQ, foi a presença de $L m$ na placenta, identificada pela forma baciliforme, apresentação característica em forma da letra "V" e coloração marrom em contraste com as estruturas azuladas adjacentes (Figura 1). A observação das lâminas ao microscópio foi realizada com ampliações de 10, 40 e 100.

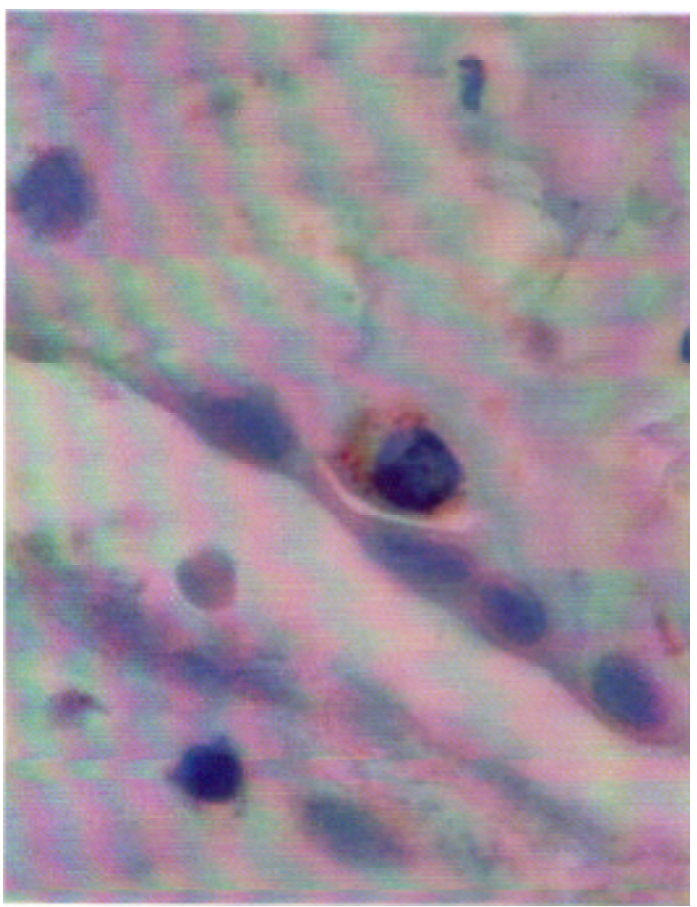

Figura 1 - Microfotografia mostrando a lesão causada pela Listeria monocytogenes na placenta, com a bactéria dentro da célula de Hofbauer e no estroma do vilo. A forma em "V" da bactéria aparece corada de marrom, em contraste às estruturas adjacentes. (IHQ- anticorpo anti-listeria; X1000). 
A consulta aos prontuários permitiu pesquisar o trimestre gestacional, a idade da paciente, o número de abortos e os exames solicitados; porém, quando a paciente foi atendida na emergência, os dados em geral eram incompletos. Em apenas um dos casos, encontramos referência sobre o resultado da cultura laboratorial da placenta com isolamento de $\mathrm{Lm}$, que foi confirmado pela IHQ.

Para a análise estatística, aplicamos o teste do $\chi^{2}$ para as variáveis alterações histológicas, trimestre gestacional, idade das gestantes, casos de aborto e/ou parto prematuro e a ocorrência de aborto habitual.

As lâminas utilizadas no presente estudo foram identificadas por números, resguardando a identidade das pacientes, e o projeto foi aprovado pelo Comitê de Ética em Pesquisa do Hospital de Clínicas de Porto Alegre, sob número 00379.

\section{Resultados}

A $L m$ foi identificada em 50 das 148 lâminas analisadas pelo método de IHQ (33,78\%).

$\mathrm{Na}$ Tabela 1, são mostradas as alterações histológicas, observadas com $\mathrm{HE}$, e a freqüência com que ocorreram nos casos positivos e negativos. A corioamnionite $(p=0,035)$ e a vilite $(p=0,003)$ mostraram ter associação significativa com a presença de $L m$ na placenta. A presença de $L m$ foi constatada em placentas de primeiro $(37,5 \%)$, segundo $(27,5 \%)$ e terceiro $(35 \%)$ trimestre gestacional, sem diferença estatística significativa $(p=0,987)$.

Tabela 1 - Freqüência de alterações histológicas nas placentas relacionadas com a presença ou ausência de $L m$ identificada pela IHQ.

\begin{tabular}{|c|c|c|c|c|}
\hline \multirow[t]{2}{*}{$\begin{array}{l}\text { Alterações } \\
\text { histológicas* }\end{array}$} & \multicolumn{2}{|c|}{$\begin{array}{l}\text { Presença de } L m \\
\quad(n=50)\end{array}$} & \multicolumn{2}{|c|}{$\begin{array}{c}\text { Ausência de } L m \\
\quad(\mathrm{n}=98)\end{array}$} \\
\hline & $\mathrm{n}$ & $\%$ & $\mathrm{n}$ & $\%$ \\
\hline Corioamnionite & 13 & 26,0 & 12 & 12,2 \\
\hline Vilite & 13 & 26,0 & 8 & 8,1 \\
\hline Deciduite & 7 & 14,0 & 14 & 14,2 \\
\hline Hemorragia & 40 & 80,0 & 80 & 81,6 \\
\hline Necrose & 12 & 24,0 & 28 & 28,5 \\
\hline Trombose & 7 & 14,0 & 10 & 14,2 \\
\hline
\end{tabular}

*Em algumas placentas, visualizou-se mais de um elemento histológico.

A idade das gestantes variou entre 17 e 48 anos, sem diferença estatística significativa entre casos positivos e negativos. Na Tabela 2, são mostrados os casos de aborto e parto prematuro e a freqüência com que ocorreram, relacionando-os com a presença ou ausência de $L m$ na placenta. Não houve associação entre a ocorrência de aborto e parto prematuro $(\mathrm{p}=0,445)$ nas duas circunstâncias (presença ou ausência de $L m$ ). Porém, quando $L m$ estava presente nas placentas, ocorreram $66,6 \%$ de abortos e $33,3 \%$ de partos prematuros nos casos em que se obteve esta informação $(n=36)$.

Tabela 2 - Freqüência de aborto, parto prematuro e nascimento a termo relacionados com a presença ou ausência de $L m$ identificada na placenta pela IHQ.

\begin{tabular}{lcccc} 
Casos & $\begin{array}{c}\text { Presença de } \mathbf{L m} \\
(\mathbf{n}=\mathbf{5 0 )}\end{array}$ & \multicolumn{2}{c}{$\begin{array}{c}\text { Ausência de } \mathbf{L m} \\
(\mathbf{n}=\mathbf{9 8})\end{array}$} \\
& $\mathbf{n}$ & $\%$ & $\mathbf{n}$ & $\%$ \\
\hline Aborto & 24 & 33,3 & 48 & 66,6 \\
Parto prematuro & 12 & 41,3 & 17 & 52,6 \\
Nascimento a termo & 0 & - & 2 & 100,0 \\
Não informado & 14 & 31,1 & 31 & 68,8 \\
\hline
\end{tabular}

Os casos de aborto habitual ou de repetição ocorreram com a presença de $\operatorname{Lm}(20,8 \%)$ ou ausência de $L m(16,6 \%)$, sem diferença significativa $(p=0,665)$, como mostrado na Tabela 3. Porém, $L m$ estava presente em $38,4 \%$ do total de casos de aborto habitual.

Tabela 3 - Ocorrência de aborto habitual relacionado com a presença ou ausência de Lm identificada na placenta pela $I H Q$.

\begin{tabular}{|c|c|c|c|c|c|}
\hline \multirow{2}{*}{$\begin{array}{l}\text { Aborto } \\
\text { habitual }\end{array}$} & \multicolumn{2}{|c|}{ Presença de $L m$} & \multicolumn{2}{|c|}{ Ausência de $L m$} & \multirow[t]{2}{*}{ Total } \\
\hline & $n$ & $\%$ & $n$ & $\%$ & \\
\hline Sim & 5 & 20,8 & 8 & 16,6 & 13 \\
\hline Não & 19 & 79,1 & 40 & 83,3 & 59 \\
\hline Total & 24 & 100,0 & 48 & 100,0 & 72 \\
\hline
\end{tabular}

\section{Discussão}

O presente estudo identificou $L m$ em $33,7 \%$ das placentas analisadas pela IHQ. Somente uma das placentas tinha a cultura e o isolamento de Lm confirmado, pois este exame não é realizado como rotina no HCPA. Todas as sete placentas examinadas no estudo desenvolvido por Parkash et al. ${ }^{11}$ apresentaram resultado positivo pela IHQ, confirmando o diagnóstico anterior realizado por meio da cultura e isolamento de $\mathrm{Lm}$.

Os resultados deste trabalho mostram que a corioamnionite e a vilite estão presentes na placenta com $\mathrm{Lm}$, concordando com os resultados encontrados por Parkash et al. ${ }^{11}$. Concordam também com o observado por Gersell e $\operatorname{Kraus}^{14}$, que afirmam que a $L m$ é a bactéria que mais comumente causa vilite, na qual predominam os 
neutrófilos, que se localizam entre o trofoblasto e o estroma dos vilos. A corioaminionite é usualmente grave e muitas vezes se estende até o estroma placentário e o âmnion contém um grande número de microrganismos.

A maioria dos autores refere que a listeriose predomina no terceiro trimestre gestacional, ocorre raramente no segundo e excepcionalmente no primeiro ${ }^{11,15}$ Em trabalhos similares, analisando placentites por $\mathrm{Lm}$ em período perinatal com detecção por IHQ ou mediante cultura e isolamento do microrganismo, pode-se observar que a infecção por $L m$ ocorreu mais no final da gestação. Efetivamente, quando a cultura é realizada para diagnóstico clínico presuntivo, pode-se encontrar este agente. Porém, culturas não são rotineiramente realizadas em abortos espontâneos e a ausência de estudo retrospectivo como o desta pesquisa pode ter contribuído para a observação da diferença dos resultados encontrados por esses autores. Esta pesquisa examinou o material proveniente dos três trimestres gestacionais e foi possivel detectar $\mathrm{Lm}$ em todos.

Esse fato não parece ser, no entanto, um fator importante para as seqüelas da listeriose, conforme observado neste estudo, ressaltando-se que o uso de IHQ evidenciou que esta infecção pode estar presente no primeiro trimestre. Ocorre que este diagnóstico não é investigado, na maioria das vezes, pela situação clínica da paciente que busca atendimento no setor de emergências dos hospitais e nem sempre está realizando acompanhamento pré-natal ou pesquisa de infertilidade.

No estudo realizado por Parkash et al. ${ }^{11}$, a idade das pacientes variou entre 21 e 41 anos. Confirmando os achados deste trabalho, a idade materna parece estar relacionada com o período de maior fertilidade da mulher mas não ter relação com a patogenicidade da $\mathrm{Lm}$.

Salazar et al. ${ }^{16}$ afirmam que não há evidências de que $L m$ cause aborto habitual, porém os resultados deste trabalho mostram que o microrganismo estava presente na placenta de cinco dos 13 casos de aborto habitual analisados $(38,4 \%)$.

A realização deste estudo permitiu identificar facilmente a presença de $L m$ em placentas que apresentavam vilite e corioamnionite, indicando que a IHQ pode ser utilizada para confirmar o diagnóstico de listeriose quando o exame de rotina da placenta evidenciar este tipo de alteração.

A alta freqüência dessa positividade $(33,78 \%)$ em tecido placentário com alterações inflamatórias nos estimula a incentivar a busca deste agente por meio da IHQ, a fim de confirmar a presença do patógeno, notadamente em casos de aborto habitual.
Mais pesquisas utilizando a técnica de IHQ poderão esclarecer a freqüência de $L m$ como uma das causas de septicemia neonatal, de aborto e parto prematuro ${ }^{17}$ e as evidências de que cause aborto habitual ${ }^{16}$.

\section{ABSTRACT}

Purpose: to identify Listeria monocytogenes (Lm) in human placentas by immunohistochemistry (IHC) and relate its presence to the histological alterations found on conventional examination, to the pregnancy trimester, age of pregnant women, cases of abortion and premature delivery, and to the occurrence of habitual abortion.

Methods: a retrospective study was carried out at the pathology service of a teaching hospital in the city of Porto Alegre in 2000. The paraffin blocks of 254 placentas, obtained from abortion, premature delivery and full-term birth, were analyzed by conventional histology using hematoxylin and eosin (HE) staining. The IHC assay consisted of a rabbit anti-listeria polyclonal antibody B65420R $\left(\right.$ Biodesign ${ }^{\circledR}$ ) diluted 1:1000, in addition to the avidin-biotinstreptavidin complex; 148 placentas revealed inflammatory disorders, hemorrhage, necrosis and thrombosis. The $\chi^{2}$ test was used for statistical analysis.

Results: Listeria monocytogenes was detected in $33.78 \%$ of the placentas analyzed by IHC. Chorioamnionitis and villitis showed significant statistical difference in the positive placentas. Lm occurred in the 1st, 2nd and 3rd trimester of pregnancy. The age of pregnant women, the cases of abortion and/or premature births were not statistically different as to the presence or absence of $\mathrm{Lm}$ in the placentas. Habitual abortions occurred in patients with or without $\mathrm{Lm}$ in the placental tissue.

Conclusion: Immunohistochemistry may be used to confirm the histopathological diagnosis of listeriosis in all trimesters of pregnancy.

KEYWORDS: Placenta. Listeria monocytogenes. Habitual abortion. Chorioamnionitis.

\section{Agradecimentos}

Aos técnicos Flavia Giustti, Jorge Alberto Lopes e Neiva Copetti do Laboratório de Patologia Experimental do HCPA; ao Fundo de Incentivo à Pesquisa e Eventos do HCPA, pelo apoio financeiro. 


\section{Referências}

1. Iida T, Kanzaki M, Nakama A, Kokubo Y, Maruyama $\mathrm{T}$, Kaneuchi, C. Detection of Listeria monocytogenes in humans, animals and foods. J Vet Med Sci 1998; 60:1341-3.

2. Limaye AP, Perkins JD, Kowdley KV. Listeria infection after liver transplantation: report of a case and review of the literature. Am J Gastroenterol 1998; 93:1942-4.

3. Slutsker L, Altekruse SF, Swerdlow DL. Foodborne diseases. Emerging pathogens and trends. Infect Dis Clin North Am 1998; 12:199-216.

4. Hofer E, Nascimento RS, Oliveira MA. Meningite por Listeria monocytogenes. Relato de casos em pacientes do Distrito Federal. Rev Soc Bras Med Trop 1998; 31:173-7.

5. Nolla-Salas J, Almela M, Gasser I, Latorre C, Salvado M, Coll P. Spontaneous Listeria monocytogenes peritonitis: a population-based study of 13 cases collected in Spain. Am J Gastroenterol 2002; 97:1507-11.

6. Laciar AL, Hasuoka RP, Correa SM, Miranda AM, Centorbi ONO. Symptomatic hydrocephalus in a newborn infected with Listeria monocytogenes. Braz J Microbiol 2000; 31:9-11.

7. Outbreak of listeriosis associated with homemade Mexican-style cheese -North Carolina, October 2000-January 2001. MMWR Morb Mortal Wkly Rep. 2001; 50:560-2.

8. Berzioli M, Brisotto P, Breseghello S, Faion N, Boccato S. Perinatal listeriosis. Apropos of two cases. Pediatr Med Chir 1999; 21:39-41.
9. Loguercio AP, Silva WP, Aleixo JAG, Costa MM, Vargas AC. Listeria monocytogenes: um importante patógeno de origem alimentar. Hig Aliment 2001; $15: 39-48$

10.Di Maio H. Listeria infection in women. Prim Care Update Ob Gyns 2000; 7:40-5.

11.Parkash V, Morotti RA, Joshi V, Cartun R, Rauch CA, West AB. Immunohistochemical detection of Listeria antigens in the placenta in perinatal listeriosis. Int J Gynecol Pathol 1998; 17:343-50.

12.Schwab JP, Edelweiss MIA. Identificação de Listeria monocytogenes em placentas humanas e espécimes de aborto pela técnica de imunohistoquimica. J Bras Patol Med Lab 2003; 39:111-4.

13.Santos RTM, Wakamatsu A, Kanamura CT, Nonogaki $\mathrm{S}$, Pinto GA. Procedimentos laboratoriais em imunohistoquímica e hibridização "in situ". In: Alves VAF, Bacchi CE, Vassalo J, editores. Manual de ImunoHistoquímica. $1^{\mathrm{a}}$ ed. São Paulo: Sociedade Brasileira de Patologia; 1999. p.237-59.

14.Gersell DJ, Kraus FT. Diseases of the placenta. In: Kurman RJ, editor. Blaustein's Pathology of the Female Genital Tract. $5^{\text {th }}$ ed. New York: Springer; 2002. p.1119-31.

15.Smith JL. Foodborne infections during pregnancy. J Food Prot 1999; 62:818-29.

16.Salazar CC, Cunha Filho JSL, Schlatter D, et al. Abortamento de repetição. Femina 2001; 29:667-72.

17.Cunningham FG, MacDonald PC, Gant NF, Leveno KJ, Gilstrap III LC. Williams Obstetrics. $20^{\text {th }}$ ed. East Norwalk: Appleton \& Lange; 1997. p.1448.

Recebido em: 22/2/2003 Aceito com modificações em: 31/7/2003

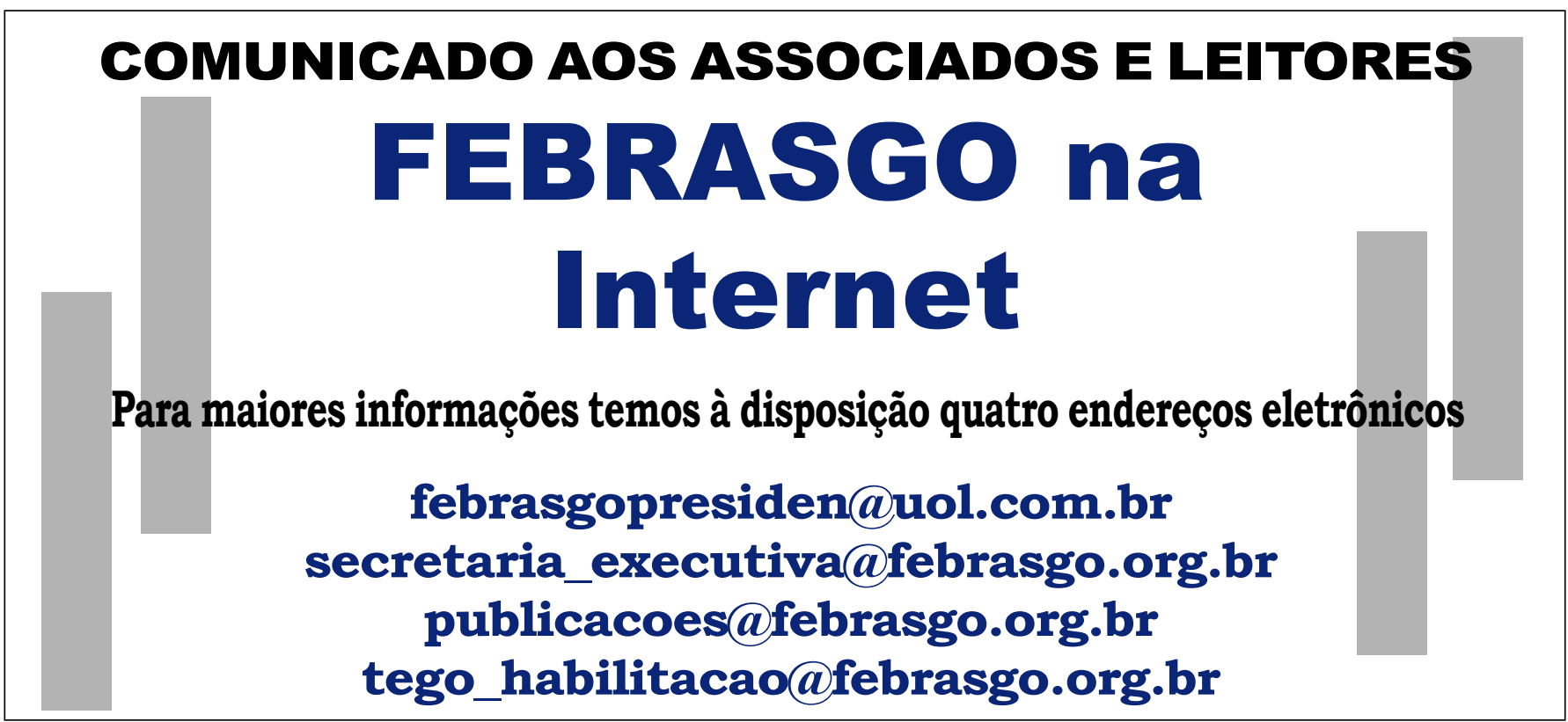

